\title{
Resonant coupling of collective intra- and intersubband excitations in a parabolically confined electron system
}

\author{
M. Kaloudis, K. Ensslin, and A. Wixforth \\ Sektion Physik, Universität München, D-8000 München 22, Germany \\ M. Sundaram, J. H. English, and A. C. Gossard \\ Materials Department, University of California, Santa Barbara, California 93106
}

(Received 28 July 1992)

\begin{abstract}
A wide electron system as realized in modulation-doped parabolic quantum wells is used to investigate the resonant interaction between intra- and intersubband plasmonic excitations. In contrast to a conventional quasi-two-dimensional electron system, both excitations have about the same resonance energy at finite wave vector due to a relatively low subband spacing. We use a perpendicular magnetic field to tune the intrasubband plasmon such that it energetically degenerates with the intersubband mode. In the electrical quantum limit no mode coupling is observed, whereas for the case of two occupied subbands a strong resonant interaction of both modes is reflected in anticrossing behavior.
\end{abstract}

Electron systems in wide remotely doped parabolic quantum wells (PQW's) have recently attracted considerable interest both experimentally and theoretically. They reveal an attempt to realize the theoretical construct of jellium, consisting of a highly mobile dilute electron gas in the potential of a positively charged background. Far-infrared spectroscopy ${ }^{1-3}$ on such structures yielded in the formulation of the generalized Kohn's theorem 4 and thus has a large impact also on the understanding of the infrared response of harmonically bound quasi-onedimensional quantum wires or quasi-zero-dimensional quantum dots. It states that in an external parabolic confining potential far-infrared radiation (FIR) only couples to the motion of the center of mass of the electron system independent of electron-electron interaction, resulting in a single line in the absorption spectra. This resonance frequency $\omega_{0}$ is then given by construction by the three-dimensional plasma frequency of a fictitious charge $n^{+}$, corresponding to the curvature $\partial^{2} E_{C} / \partial z^{2}$ of the bare confining potential:

$$
\omega_{0}^{2}=\frac{e^{2} n^{+}}{\epsilon \epsilon_{0} m^{*}}, \quad n^{+}=\frac{\epsilon \epsilon_{0}}{e^{2}} \frac{\partial^{2} E_{C}}{\partial z^{2}} .
$$

Here $\epsilon$ is the dielectric constant of the background, whereas the other symbols have their usual meaning. Advances in growth processes made possible to intentionally shape the conduction- and valence-band edges of $\mathrm{Al}_{x} \mathrm{Ga}_{1-x} \mathrm{As}$ quantum wells by appropriately adjusting the aluminum fraction $x$ of the alloy. ${ }^{5}$ Hence the infrared response of such systems can be tailored in a very unique manner. In a recent experiment, we were able to show that it is possible to excite the intersubbandlike plasmon mode by means of a grating coupler technique ${ }^{6}$ and to study the effect of a variable layer thickness or equivalently variable carrier density on the observed resonance.

Here we present the results of an experiment, where by proper choice of the grating coupler ${ }^{7}$ we can simultaneously excite both intrasubband plasmon excitations as well as the intersubbandlike mode $\omega_{0}$ and study their interaction. The resonant interaction of surface plasmons and intersubband resonances has first been calculated by Das Sarma ${ }^{8}$ employing a random-phase-approximation frame in a twoband model, and later by $\mathrm{Li}$ and Das Sarma, ${ }^{9}$ and by Gold and Ghazali. ${ }^{10}$ Experimentally, it has been observed before by Oelting, Heitmann, and Kotthaus $^{11}$ for the case of a two-dimensional electron system (2DES) on Silicon-MOS structures. For a 2DES confirmed in a triangular potential well, however, usually the intersubband-resonance energies are much larger than those of intrasubband plasmons for experimentally accessible grating periods and carrier densities such that the coupling is only a second-order effect. By application of uniaxial stress, however, the authors of Ref. 11 succeeded in tuning and energetically matching the resonance frequencies. Consequently, they observed resonant interaction between the two excitations and obtained qualitative agreement with the calculation of Das Sarma. ${ }^{8}$ In our experiment the intersubband-resonance energy is much smaller than in a 2DES due to the large width of the confining potential in a PQW. ${ }^{12}$ Typical curvatures of the samples studied in our experiments result in $\hbar \omega_{0}$ in the range $20-80 \mathrm{~cm}^{-1}$. The surface (intrasubband) plasmon dispersion, however, is expected to behave as in the 2 DES case, ${ }^{13,14}$ i.e.,

$$
\omega_{P}^{2}=e^{2} n_{s} q / 2 \bar{\epsilon} m_{P},
$$

where $n_{s}$ denotes the areal carrier density, $q=(2 \pi / a)$ the wave vector of the grating coupler of periodicity $a, \bar{\epsilon}$ an effective dielectric function, and $m_{p}$ the plasmonic effective mass. For a carrier density of approximately $3 \times 10^{11} \mathrm{~cm}^{-2}$ and a grating coupler periodicity of $2 \mu \mathrm{m}$ the intrasubband-plasmon energy $\hbar \omega_{P}$ is of the order of $20 \mathrm{~cm}^{-1}$, thus comparable to the one of the intersubbandlike mode.

The experiment presented here is done on a $200-\mathrm{nm}$ wide, 150-meV-deep PQW with additional vertical 75meV-high sidewalls. The curvature of the parabolic well 
thus leads to an expected resonance frequency of $\hbar \omega_{0}=46$ $\mathrm{cm}^{-1}=5.7 \mathrm{meV}$. By application of a negative bias between a semitransparent $\mathrm{NiCr}$ gate electrode and the electron system we are able to tune the carrier density $n_{s}$, the number of occupied subbands, and within certain limits also the shape of the confining potential. ${ }^{6,15}$ At $V_{g}=0$ $V$ the carrier density is $n_{s}=2.3 \times 10^{11} \mathrm{~cm}^{-2}$ with two subbands occupied. At $V_{g} \approx-0.3 \mathrm{~V}$ the second subband depopulates and at $V_{g}=-1.3 \mathrm{~V}$ the PQW becomes completely depleted. At $V_{g}=0 \mathrm{~V}$ and $T=4 \mathrm{~K}$ the electron mobility is of the order of $\mu=25 \mathrm{~m}^{2} \mathrm{~V}^{-1} \mathrm{~s}^{-1}$.

Our measurements are carried out using a rapid-scan Fourier spectrometer, with the sample placed in a superconducting solenoid $\left(B_{\max }=15.5 \mathrm{~T}\right)$ held at $T=2 \mathrm{~K}$. Experimentally, we determine the relative change in transmission $-\Delta T / T=\left[T(0)-T\left(n_{s}\right)\right] / T(0)$ of the FIR, which is proportional to the real part of the dynamic conductivity $\sigma(\omega, B) .{ }^{16}$ A silver grating coupler of periodicity $a=2 \mu \mathrm{m}$ is placed on top of the semitransparent gate electrode, thus providing both the necessary $z$ component of the FIR field to couple to the intersubbandlike mode $\omega_{0}$ as well as the wave vector $q=2 \pi / a$ parallel to the surface to couple to the intrasubband plasmon. ${ }^{17}$ From that we expect the plasmon resonance to occur at $\hbar \omega_{P} \approx 19 \mathrm{~cm}^{-1}=2.36 \mathrm{meV}$ for $V_{g}=0 \mathrm{~V}$ if we use an effective dielectric constant of $\bar{\epsilon}=13$ and $m_{p}=0.07 m_{0}$. Since there is a small uncertainty for the actual curvature of a PQW for a given sample, we determine $\omega_{0}$ independently for the same sample but before the preparation of the grating employing tilted field experiments ${ }^{3}$ at $q=0$, resulting in $\hbar \omega_{0}=44 \pm 2 \mathrm{~cm}^{-1}$. We wish to point out, however, that for $q \neq 0$ a strict separation of the modes is no longer possible, since for a finite width of the slab both can have both surface and bulk character, respectively. ${ }^{9,14}$

For a given $q$ in principle $\omega_{p}$ can be tuned via its $n_{s}$ dependence. This dependence is indeed observed on our samples, but it is not possible to energetically degenerate $\omega_{p}$ and $\omega_{0}$ for any of the samples studied. Instead we use a perpendicular magnetic field $B$ to tune the intrasubband plasmon energy. Here the effect of the cyclotron motion on the surface plasmon leads to the well-known expression for the magnetoplasmon ${ }^{13}$ excitation $\omega_{\mathrm{MP}}^{2}=\omega_{C}^{2}+\omega_{P}^{2}$, with $\omega_{c}=e B / m^{*}$ the cyclotron frequency.

In Fig. 1 we present a typical set of spectra as obtained experimentally for a gate bias of $V_{g}=0 \mathrm{~V}$. In this case two electric subbands are occupied. We depict the relative change in transmission $-\Delta T / T$ as a function of energy for different magnetic-field values $B$. For low magnetic fields one observes three absorption lines, which we identify as cyclotron-resonance (CR), intrasubbandmagnetoplasmon (MP), and intersubbandlike plasmon (ISR), respectively. With increasing magnetic field both the CR and the MP shift to higher energies according to the expected magnetic-field dispersion. For $B \approx 2.8 \mathrm{~T}$, however, a strong resonant interaction of MP and ISR is observed, reflecting in an anticrossing behavior.

Following the initial two-level theory of Das Sarma ${ }^{8}$ and using his terms, interaction of the surface (intrasubband) -plasmon $\omega_{p}$ and the intersubband mode $\omega_{21}$ leads to an expression for the coupled modes:

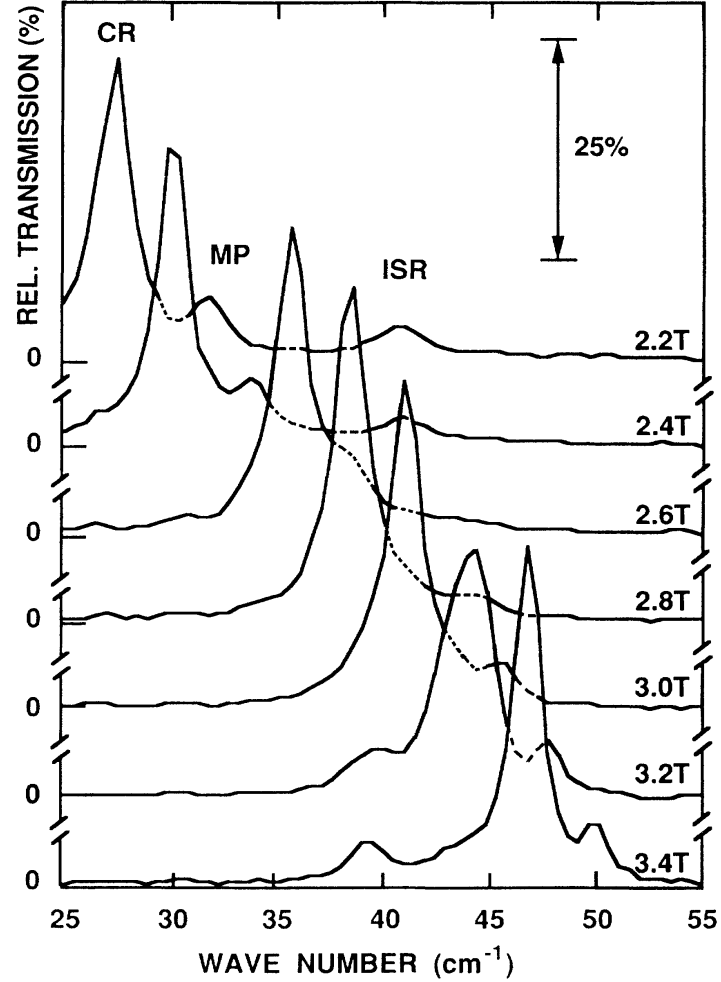

FIG. 1. Typical set of spectra as obtained on a 200-nm-wide PQW with a $2-\mu \mathrm{m}$ grating coupler. We plot the relative change in transmission as a function of the FIR energy for different magnetic fields. The spectra have been vertically offset for clarity. At low as well as for high magnetic fields three lines are observed which are interpreted in terms of cyclotron resonance (CR), intrasubband-magnetoplasmon (MP), and intersubband plasmon (ISR). Around $B \approx 2.8 \mathrm{~T}$ the intra- and intersubband modes interact resonantly, whereas the $C R$ remains unaffected.

$$
\omega_{ \pm}^{2}=\frac{1}{2}\left\{\left(\omega_{21}^{2}+\omega_{P}^{2}\right) \pm\left[\left(\omega_{21}^{2}-\omega_{P}^{2}\right)^{2}+4 \omega_{i}^{4}\right]^{1 / 2}\right\} .
$$

For a two-dimensional space-charge layer $\omega_{21}=\left(E_{21}^{2}+W_{P}^{2}\right)^{1 / 2}$ denotes the depolarization shifted intersubband resonance frequency with $W_{P}$ $=\left[2 n_{S} E_{21} v_{1212}(q \rightarrow 0)\right]^{1 / 2}$. The frequency

$$
\omega_{i}^{2}=q\left\{2 n_{S}^{2} E_{21}\left[v_{1112}(q \rightarrow 0)\right]^{2} / m\right\}^{1 / 2}
$$

determines the strength of the coupling, where $E_{21}$ is the self-consistent subband spacing and $v_{i j k l}$ the matrix elements for Coulomb interaction between the modes $i j$ and $k l$ in subbands 1 and 2, respectively. For a symmetric potential well this matrix element turns out to be strictly zero for arbitrary $q$ if $(i+j+k+l)$ is an odd number. Therefore, the two-band model predicts no mode coupling to occur for our PQW. If, however, more occupied electrical subbands are taken into account, this is no longer true. Employing a three-band model, Li and Das Sarma have shown ${ }^{9}$ that in this case the mode coupling indeed recovers due to the interaction of the intrasubband excitation in subbands 1 and 2 with the intersubband excitation between subband 2 and 3 . Since we are tuning the intrasubband plasmon via the magnetic field as described above, $\omega_{p}$ in Eq. (3) has to be replaced by $\omega_{\mathrm{MP}}$, leading to 

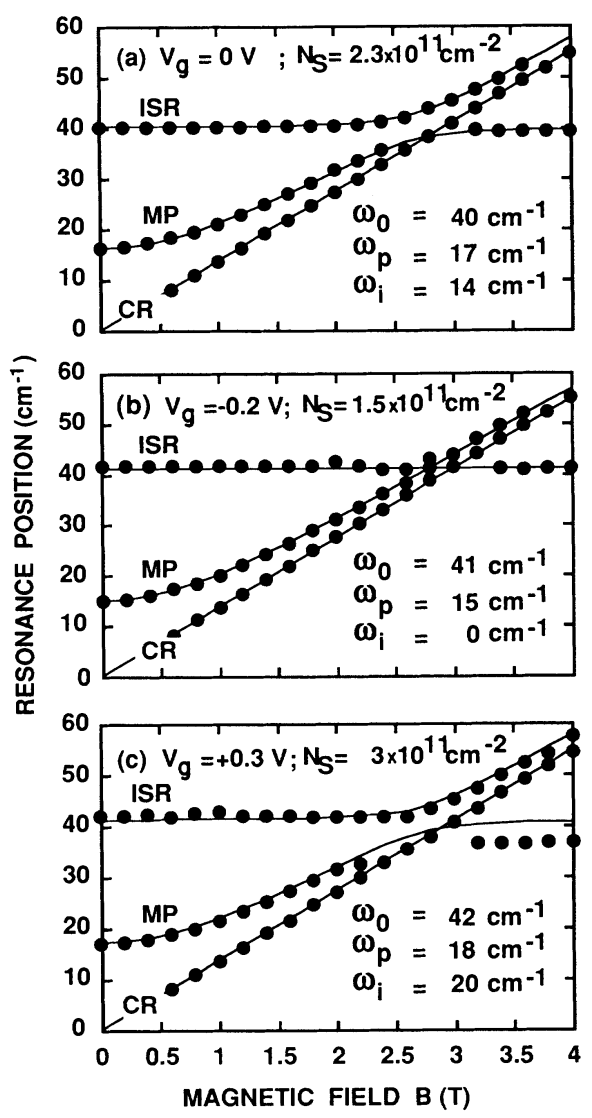

FIG. 2. (a) Resonance positions as obtained from the spectra of Fig. 1. The dots mark the experimental result, whereas the lines are the result of the calculations involving Das Sarma's two-band model for all three modes. Clearly a resonant coupling of the intra- and intersubbandlike modes is observed and results in an anticrossing of the two modes. To obtain the best agreement between theory and experiment, the parameters as listed in the inset have been used. (b) The same as in (a) but for a lower gate bias or equivalently a smaller number of electrons in the well. In this case the second subband is barely occupied. As compared to (a), the mode coupling is considerably reduced if not quenched at all. (c) For comparison, the same result for a more positive gate bias corresponding to a higher electron concentration with at least three occupied subbands. In this case the mode coupling is strongly enhanced as compared to (a) and (b) and the two-band model does not hold any longer.

$$
\begin{aligned}
\omega_{ \pm}^{2}=\frac{1}{2} & \left(\left[\omega_{0}^{2}(q)+\omega_{\mathrm{MP}}^{2}\left(q, n_{S}, B\right)\right]\right. \\
& \left. \pm\left\{\left[\omega_{0}^{2}(q)-\omega_{\mathrm{MP}}^{2}\left(q, n_{S}, B\right)\right]^{2}+4 \omega_{i}^{4}\right\}^{1 / 2}\right) .
\end{aligned}
$$

The result is shown in Fig. 2(a), where we plot it together with the observed resonance positions for all modes as a function of the magnetic field $B$. Special care was taken not to tilt the magnetic field with respect to the normal of the PQW layer, as this leads to an additional coupling of the CR and the ISR mode ${ }^{1,3}$ which would unnecessarily complicate the study presented here. No such signature is observed in our data, hence we are sure that the resonant anticrossing between MP and ISR is solely determined by the Coulomb coupling of both modes. Using the two-band model for simplicity, the coupling is ex- pressed in terms of $\hbar \omega_{i}=14 \mathrm{~cm}^{-1}=17.4 \mathrm{meV}$ to obtain optimum agreement with our data. The grating coupler induced intersubband resonance in this case has due to its $q$ dependence a somewhat lower value than the one we extract from $q=0$ experiments on the same sample, namely $\omega_{0}(q) \approx 41 \mathrm{~cm}^{-1}$.

In Fig. 2(b) we present the experimental result for a gate bias of $V_{g}=-0.2 \mathrm{~V}$. In this case we approach the electrical quantum limit, i.e., only one subband remaining occupied. In terms of the two-band model no mode coupling is expected to occur under this condition. This prediction is indeed verified in our experiment. Although we cannot state definitely that there is no coupling at all due to the resolution of our experiment, the anticrossing gap is clearly reduced in comparison to Fig. 2(a). Selfconsistent calculations ${ }^{6,15}$ confirm that the confining potential under this condition is still rather symmetric, such that the two-band model as employed above should hold. However, since the model of Refs. 8 and 9 is derived for an infinite rectangular quantum well and in a PQW the confining potential is harmonic, we cannot give a simple quantitative comparison of our experiment and the existing theories. In our case, however, the generalized Kohn theorem holds and thus should simplify a future theoretical description by a considerable amount. Also, the influence of the small but finite perpendicular magnetic field on the coupling is not known, to date. We have not yet been able to recover the predicted mode coupling by breaking the symmetry of the PQW potential by application of a larger negative bias. ${ }^{6}$ This should occur, since in a nonsymmetric potential the matrix element $v_{1112}$ is not necessarily zero. ${ }^{9}$ Such studies, however, are presently being carried out and will be the subject of future investigations. For comparison, we depict in Fig. 2(c) the same results for a more positive gate bias and consequently a larger number of electrons an electrical subbands in the well. Here at least three occupied subbands are involved and the mode coupling is strongly enhanced as compared to cases (a) and (b). As expected, in this case a description in terms of the two-band model is no longer possible.

In summary, we observe resonant interaction between the basic collective excitations of a quasijellium layer at finite $q$. These modes are identified with an intrasubband and an intersubband plasmon. To cause both modes to become energetically degenerate, we use a perpendicular magnetic field and tune the intrasubband plasmon energy according to the magnetoplasmon dispersion. The resonant interaction leads to a strong anticrossing and mixing of both modes as a function of the magnetic field. In the electrical quantum limit, however, the symmetry of the confining potential seems to quench this mode coupling, as is predicted by a recent theory. ${ }^{9}$

We gratefully acknowledge very enlightening discussion with Jörg P. Kotthaus, Sankar Das Sarma, and Edwin Batke, and thank Stephan Manus and Stefan Kühn for technical assistance. This work has been sponsored in part by the U.S. Air Force Office of Scientific Research under Contract No. AFO SR-88-099 and in part by the Deutsche Forschungsgemeinschaft (DFG). 
${ }^{1}$ K. Karrai, H. D. Drew, H. W. Lee, and M. Shayegan, Phys. Rev. B 39, 1426 (1989).

${ }^{2}$ K. Karrai, X. Ying, H. D. Drew, and M. Shayegan, Phys. Rev. B 40, 12020 (1989).

${ }^{3}$ A. Wixforth, M. Sundaram, J. H. English, and A. C. Gossard, Surf. Sci. 267, 523 (1992).

${ }^{4}$ L. Brey, N. F. Johnson, and B. I. Halperin, Phys. Rev. B 40, 10647 (1989).

${ }^{5}$ M. Sundaram, A. C. Gossard, J. H. English, and R. M. Westervelt, Superlatt. Microstruct. 4, 683 (1988).

${ }^{6}$ A. Wixforth, M. Sundaram, J. H. English, and A. C. Gossard, Phys. Rev. B 43, 10000 (1991).

${ }^{7}$ E. Batke, G. Weimann, and W. Schlapp, Phys. Rev. B 39, 11171 (1989).

${ }^{8}$ S. Das Sarma, Phys. Rev. B 29, 2334 (1984).

${ }^{9}$ Q. Li and S. Das Sarma, Phys. Rev. B 40, 5680 (1989).

${ }^{10}$ A. Gold and A. Ghazali, Phys. Rev. B 41, 8318 (1990).

${ }^{11}$ S. Oelting, D. Heitmann, and J. P. Kotthaus, Phys. Rev. Lett. 56, 1846 (1986).
${ }^{12}$ For two-dimensional hole space-charge layers, in principle energetically low-lying intersubband resonances exist, which may become degenerate with the intrasubband plasmons. Due to the large linewidths, however, no such coupling has been observed. A. D. Wieck, E. Batke, D. Heitmann, and J. P. Kotthaus, Surf. Sci. 142, 442 (1984).

${ }^{13}$ A. V. Chaplik, Zh. Eksp. Teor. Fiz. 62, 746 (1972) [Sov. Phys. JETP 35, 395 (1972)].

14J. Dempsey and B. I. Halperin, Phys. Rev. B 45, 1719 (1992). In fact, this behavior has recently been observed in parabolic quantum wells by P. R. Pinsukanja, E. Yuh, E. L. Asmar, E. G. Gwinn, M. Sundaram, and A. C. Gossard, Phys. Rev. B 46, 7284 (1992).

${ }^{15}$ A. Wixforth, M. Sundaram, K. Ensslin, J. H. English, and A. C. Gossard, Appl. Phys. Lett. 56, 454 (1990).

${ }^{16}$ E. Batke and D. Heitmann, Infrared Phys. 24, 189 (1984).

${ }^{17}$ See, e.g., L. Zheng, W. L. Schaich, and A. H. MacDonald, Phys. Rev. B 41, 8493 (1990). 